\title{
Changes to the World Health Organization guideline on hormonal contraceptive eligibility for women at high risk of HIV: South African perspective and response
}

\author{
Z Nene, ${ }^{1}$ BSc, MB ChB, Dip Palliative Med, DOH, MMed (O\&G), FCOG (SA), Cert Reproductive Medicine (SA); \\ G J Hofmeyr, ${ }^{2}$ MB BCh, MRCOG, DSc; M Patel, ${ }^{3}$ MB ChB, FCOG (SA), MMed (O\&G), Cert Reproductive Medicine (SA); \\ M Panday, ${ }_{4}^{4}$ MB ChB, FCOG (SA); H Rees, ${ }^{5}$ MB BChir, MA (Cantab), MRCGP, DCH, DRCOG; M Makua, ${ }^{6}$ DLitt et Phil, MTech; Y Pillay, ${ }^{6}$ PhD \\ ${ }^{1}$ Reproductive and Endocrine Unit, Department of Obstetrics and Gynaecology, Faculty of Health Sciences, University of Pretoria and \\ Steve Biko Academic Hospital, Pretoria, South Africa \\ ${ }^{2}$ Effective Care Research Unit, Faculty of Health Sciences, University of Fort Hare, and Frere and Cecilia Makiwane Hospitals, East London, South Africa \\ ${ }^{3}$ Reproductive Medicine Unit, Department of Obstetrics and Gynaecology, Faculty of Health Sciences, University of Cape Town and \\ Groote Schuur Hospital, Cape Town, South Africa \\ ${ }^{4}$ Family Planning Department, King Dinuzulu Hospital Complex and Department of Obstetrics and Gynaecology, Nelson R Mandela School of \\ Medicine, College of Health Sciences, University of KwaZulu-Natal, Durban, South Africa \\ ${ }^{5}$ Wits Reproductive Health and HIV Institute, University of the Witwatersrand, Johannesburg, South Africa \\ ${ }^{6}$ National Department of Health, Pretoria, South Africa
}

Corresponding author: Z Nene (zozo.nene@up.ac.za)

\begin{abstract}
The World Health Organization (WHO) published guidelines for hormonal contraceptive eligibility for women at high risk of HIV in March 2017. This guidance followed from a technical consultative meeting convened by the WHO in December 2016, where all the available evidence on hormonal contraceptives and risk of HIV acquisition was reviewed. This was an expert meeting with representation from global experts in family planning and HIV management, including clinicians, epidemiologists, researchers and civil society. The guideline development group, through a consensus, made recommendations to change the medical eligibility criteria for contraceptive use from category 1 to category 2 for progestogen-only injectable contraceptives among women at high risk of HIV. There was no change in the recommendation for all other methods of hormonal contraception. The data that informed this decision are from observational studies, which have limitations; therefore, causality or association of hormonal contraception and risk of HIV acquisition have not been proven. This guidance will have an impact on countries that have a high HIV disease burden and where progestogen-only injectable contraceptives are the highest used, as in South Africa (SA). The information has to be communicated in line with the WHO's sexual and reproductive health rights principles of ensuring that all women should receive evidence-based recommendations. This will empower them to make informed choices about their reproductive needs. This article seeks to clarify the decision-making process of the WHO and how the new recommendations were formulated. It also gives SA's response to the guidance and a perspective of what informed the National Department of Health's position, taking into account the effect this will have on SA's contraceptive guidelines.
\end{abstract}

S Afr Med J 2018;108(8):629-631. DOI:10.7196/SAMJ.2018.v108i8.13160

Over the past 25 years, epidemiological and biological studies have attempted to determine whether there is an increased risk of HIV acquisition associated with the use of hormonal contraception. Until recently, all the relevant studies have been observational in design and have varied in quality. Measurement of contraceptive exposure, HIV exposure and HIV outcome was often not well controlled in these studies and there were challenges in the definition of the comparison groups. Residual or uncontrolled confounding made interpretation difficult. Sexual behaviour, condom use and contraceptive use, which were frequently self-reported, as well as bias in prescribing practices, are unmeasured confounders that could have influenced the findings of these studies. Consequently, clinical studies have disagreed on whether or not there is an increased risk of HIV acquisition with the use of hormonal methods. Furthermore, laboratory studies have been unable to clearly define the mechanisms that might drive increased HIV risk. The greatest potential concern has centred on the use of the injectable progestin depot medroxyprogesterone acetate
(DMPA). In a recent meta-analysis of observational studies, the magnitude of effect on increasing the risk of HIV acquisition was 1.40 (95\% confidence interval (CI) 1.23 - 1.59). ${ }^{[1]}$

In March 2017, the World Health Organization (WHO) released new guidance regarding injectable progestin contraceptives and the risk of HIV acquisition after a review of all the available data on hormonal methods and HIV acquisition. ${ }^{[1,2]}$ The medical eligibility criteria for contraception (MEC) categorisation for injectable progestin DMPA and norethisterone enantate (NET-EN) were changed from 1 (no restriction, with clarification) to 2 (advantages outweigh theoretical or proven risks). The combined hormonal contraceptives remained an MEC category 1 . The WHO recommends that women considering using DMPA/NET-EN should be advised that there are concerns about a possible increased risk of HIV acquisition, there is uncertainty about whether injectable contraceptive methods cause increased risk and there are ways to minimise the risk of becoming infected, such as the use of male and female condoms and pre- 
exposure prophylaxis, where available. Efforts are underway to possibly answer this question, with the first randomised controlled trial investigating whether there is a difference in risk of HIV acquisition between three different contraceptive methods. The Evidence for Contraceptive Options and HIV Outcomes (ECHO) trial is a multicentre, open-label, randomised clinical trial comparing HIV incidence and contraceptive benefits in women using injectable DMPA, the levonorgestrel (LNG) implant, and the copper intrauterine device (IUD). This study is ongoing at 12 sites in South Africa (SA), Swaziland, Zambia and Kenya and the results will be available in 2019.

\section{The World Health Organization's rationalle for the change}

The change in the WHO recommendations for hormonal contraception for women at high risk of HIV was based on the updated systematic review by Polis et al. ${ }^{[1]}$ This review included all studies published up to 15 January 2016. The data suggested a possible increase in risk of HIV acquisition in DMPA users (hazard ratio (HR) 1.40 (95\% CI 1.23 - 1.59)). The risk of NET-EN use had an HR of 1.15 (95\% CI $0.93-1.42$ ), which was not statistically significant. The authors therefore concluded that the current available data do not suggest an increased risk of HIV acquisition with the use of NET$\mathrm{EN}$. The quality of the evidence was rated as low to low-moderate. It is still unclear whether there is a real biological causal effect. Because these were observational studies, it is unknown whether the effect is due to methodological or confounding issues.

\section{Possible biological mechanism by which depot medroxyprogesterone acetate might increase HIV risk}

The proposed mechanisms by which DMPA might increase the risk of HIV acquisition are not supported by good evidence. The limitation of these studies is due to the different delivery systems of the hormones and the varied dosages.

Most are animal and laboratory studies and not validated in humans. The possible mechanisms are:

- architectural changes in female genital tract ${ }^{[2-4]}$

- epithelial thinning or disruption

- alteration in tight junctions

- alteration of adhesions

- alteration in microbiome, e.g. vaginal flora ${ }^{[3,4]}$

- enhanced HIV replication ${ }^{[5]}$

- increase in HIV susceptibility markers in the genital tract ${ }^{[5]}$

- impact on immune response ${ }^{[4,6]}$

- increased frequency of activated HIV targets cells at the cervix. ${ }^{[7]}$

\section{Implication of change in guideline}

Family planning and HIV are public health priorities; therefore, the evidence cannot be ignored. There has to be a balance of risks $\mathrm{v}$. benefits. Women have to be informed of the possible risk, but may not be denied the method if they make an informed choice to use it.

Before changing the policy and contraceptive guidelines, however, we need to be able to predict what the implications of the change will be. To address this, we have to weigh the outcomes of HIV disease burden against poor reproductive outcomes associated with unintended pregnancies, should a contraceptive method be withdrawn or withheld. The disability adjusted life-years (DALYs) are used to measure the overall burden of disease. The HIV DALYs would be the number of people who will acquire HIV and those who will die from HIV. This has to be compared with the poor reproductive outcome DALYs, i.e. maternal death and maternal morbidity due to complications of pregnancy.

If a decision is made to discontinue usage of DMPA, without replacement of a contraceptive:

- HIV DALYs will be averted owing to reduced exposure to DMPA (if a true causal association exists)

- maternal DALYs will be increased owing to reduced contraceptive usage.

If a decision is made to discontinue usage of DMPA, with full contraceptive replacement (preferably long-acting reversible contraceptive (LARC) methods):

- increased HIV DALYs will be averted (if a true causal association exists)

- low maternal adverse outcomes will occur owing to effective replacement.

There is, however, a risk of net harm if there is a low rate of replacement. There must, therefore, be willingness and an ability of women to switch contraceptive methods. This would be difficult in SA, where most women use injectable contraceptive methods.

\section{South Africa's response to the World Health Organization statement}

The evidence of association of progesterone-only injectable contraceptives and HIV acquisition is not strong. This poses a dilemma because, on the one hand, there is an obligation not to create a panic situation with resultant widespread discontinuation of injectable contraceptives and adverse health outcomes due to unintended pregnancies with increased maternal morbidity or mortality. On the other hand, DMPA is one of the most used contraceptive methods in SA, which, combined with the high burden of HIV, warrants prudent consideration and action.

On 3 April 2017, the National Department of Health initiated a meeting specifically to:

- debate the developments with regard to the current WHO contraceptive guidelines on hormonal contraceptives for women at high risk of HIV; and

- advise on the way forward for contraceptive guidelines for SA.

Therefore, in keeping with the above-mentioned mandate, the expert members' overall consensus was as follows:

- The WHO statement should be supported.

- Current SA national contraception guidelines should be upheld, but with strong emphasis on counselling and use of counselling tools.

- The uptake of LARC methods should be increased.

- There should be intensification of training and retraining of clinicians and nurses regarding the correct administration and removal of the subdermal contraceptive implant, IUDs and management of complications of these methods.

- Clinical protocols should be updated to assist primary healthcare clinicians on how to streamline referrals to higher levels of healthcare.

- The research outcome of the ECHO study, when available, should be strongly considered when reviewing the contraception guidelines. Since the study is expected to be completed by 2019 , it is only proper that the current contraception guidelines be upheld and continued to avoid unplanned and unwanted pregnancies that pose dire public and personal health consequences.

- The WHO guideline statement and the national response to the statement should be communicated to all stakeholders, academia, media, healthcare providers, the community and civil society. 


\section{Conclusion}

The National Department of Health strongly supports the rightsbased approach to the provision of sexual and reproductive health services. The literature will continually be reviewed so that evidencebased recommendations are made. More robust studies are needed to inform policy decision. We eagerly await the results of the ECHO trial. If there is upscaling of the uptake of LARC methods, SA should be ready to respond to any outcome of the study to ensure that there are no adverse HIV or maternal outcomes as a result of use or discontinuation of progesterone-only injectable contraceptives.

Recommendations for IUDs were not reviewed in this guidance. There are also no available data for the use of contraceptive patches, rings, or hormonal IUDs and risk of HIV acquisition, and very little data on implants.

\section{Acknowledgements. None.}

Author contributions. All authors made contributions to the article. ZN prepared the draft, which was approved by all authors, as was the final draft.
Funding. None.

\section{Conflicts of interest. None.}

1. Polis CB, Curtis KM, Hannaford PC, et al. An updated systematic review of epidemiological evidence on hormonal contraceptive methods and HIV acquisition in women. AIDS 2016;30(17):2665. https:/ doi.org/10.1097/QAD.0000000000001228

2. World Health Organization. Hormonal Contraceptive Eligibility for Women at High Risk of HIV Guidance Statement. Geneva: WHO, 2017. http:/who.int/reproductivehealth/publications/family planning/HC-and-HIV-2017/en/ (accessed 14 December 2017).

3. Hapgood JP, Kaushic C, Hel Z. Hormonal contraception and HIV-1 acquisition: Biological mechanisms. Endocr Rev 2018;39(1):36-78. https://doi.org/10.1210/er.2017-00103

4. Blish CA, Baeten IM. Hormonal contraception and HIV-1 transmission. Am I Reprod Immunol 2011;65(3):302-307. https://doi.org/10.1111/i.1600-0897.2010.00930.x

5. Tarleton J, Haddad L, Achilles SL. Hormonal contraceptive effects on the vaginal milieu: Microbiota and immunity. Curr Obstet Gynecol Rep 2016;5(1):20-29. https://doi.org/10.1007/s13669-016-0142-6 6. Ragupathy V, Xue W, Tan J, et al. Progesterone augments cell susceptibility to HIV-1 and HIV-1/HSV-2 co-infections. J Mol Endocrinol 2016;57(3):185-199. https://doi.org/10.1530/JME-16-0138

7. Huijbregts RP, Michel KG, Hel Z. Effect of progestins on immunity: Medroxyprogesterone bu not norethisterone or levonorgestrel suppresses the function of T cells and pDCs. Contraception 2014;90(2):123-129. https://doi.org/10.1016/j.contraception.2014.02.006

8. Byrne EH, Anahtar MN, Cohen KE, et al. Association between injectable progestin-only contraceptives and HIV acquisition and HIV target cell frequency in the female genital tract in South African women A prospective cohort study. Lancet Infect Dis 2016;16(4):441-448. https://doi.org/10.1016/S1473 3099(15)00429-6

Accepted 19 February 2018 